\title{
Efficient Method to Evaluate Critical Ricochet Angle of Projectile Penetrating into a Concrete Target
}

\author{
Min Kuk Choi $\mathbb{D}^{1},{ }^{1}$ Jihoon Han $\mathbb{D}^{2},{ }^{2}$ Sangjin Park, ${ }^{1}$ and Woo Jin An ${ }^{1}$ \\ ${ }^{1}$ The 4th R\&D Institute-3rd Directorate, Agency for Defense Development (ADD), P.O. Box 35, Yuseong-gu, \\ Daejeon 34186, Republic of Korea \\ ${ }^{2}$ Department of Mechanical Engineering, Chonbuk National University, 567 Baekje-daero, Deokjin-gu, Jeonju-si, \\ Jeollabuk-do 54896, Republic of Korea \\ Correspondence should be addressed to Min Kuk Choi; mkchoi87@add.re.kr and Jihoon Han; jihoonhan@jbnu.ac.kr
}

Received 28 June 2018; Revised 24 August 2018; Accepted 13 September 2018; Published 21 October 2018

Academic Editor: Ricardo Perera

Copyright (C) 2018 Min Kuk Choi et al. This is an open access article distributed under the Creative Commons Attribution License, which permits unrestricted use, distribution, and reproduction in any medium, provided the original work is properly cited.

In this study, we propose an efficient computation method to estimate the critical ricochet angle (CRA) for oblique penetration into concrete targets which is based on the spherical cavity-expansion theory. During penetrating event, the resistance force on the projectile nose is approximated by semi-empirical function from the spherical cavity-expansion theory and projectile motion of oblique penetration is predicted to verify the proposed numerical method with the aid of finite differential approach. In order to enhance the accuracy of projectile motion, the empirical constants of the semi-empirical function are obtained with respect to the oblique angle by conducting finite element analyses of the oblique penetration. CRA is then obtained by predicting the projectile motion at the various oblique angles and verified with results of finite element analysis. Our work presents that the reliable CRA can be estimated efficiently by employing a series of the numerical simulations. We believe that our proposed numerical method will provide a useful analysis platform for designing penetrator warhead which hits the target at an oblique impact angles.

\section{Introduction}

Since the hardened and deeply buried targets have been developed for protection of facilities in military field, penetrator warheads into concrete protective structures have attracted extensive research attention in the field of military science. Among many studies on normal impact processes, Hanchak et al. [1] and Dancygier and Yankelevsky [2,3] have been carried out normal penetration experiments of steel projectiles into concrete targets. In addition, Forrestal et al. [4] developed an semi-empirical equation, based on spherical cavity-expansion theory, to describe the penetration depth during normal penetration of ogive-nosed projectile and applied it to simulate the normal penetration process of a rigid projectile into concrete targets which are described by incompressible and linear compressible materials [5].

Generally, oblique penetration is more frequently observed than normal penetration when the penetrator warhead is designed and conducted to defeat hardened and deeply buried targets. During the impact events at oblique angles, the projectile may lead to bounce back from the target surface without penetrating or embedding the target. This phenomenon called the ricochet and especially critical oblique angle which represents the minimum oblique angle that will produce the ricochet behavior is essential to ensure the performance of the penetrator warhead. Many experimental, theoretical, and computational studies on the ricochet of various types of the projectiles and targets have been conducted at oblique angles [6-9]. Tate [6] first evaluated the ricochet of long-rod type projectiles using a simplified analytical model and extended the work [7] by modifying the analytic model to include the effect of target strength and deformation of the projectile. However, due to the physical complexity of the oblique penetration, experimental and numerical methods have been conducted to evaluate the critical ricochet angle (CRA) of long-rod type projectile only [7]. Daneshjou and Shahravi [8] calculated CRA with a full 3D explicit finite element method for various impact velocities, strength of target plates, and projectiles. In addition, studies about CRA for bullet were established 
on different target materials [9]. It is known that the ricochet depends on many factors including the impact velocity, the oblique angle, natures of both the target surface and the projectiles, the shape of projectile, etc.

However, despite numerous researches on the ricochet, CRA during the penetration into concrete targets has not been completely studied yet. When projectiles impact the concrete target with oblique angles, the target material experiences complicated compressive and/or tensile loadings which are different with that of normal impact. Thus, estimation of trajectory projectile at oblique angles is challenging research topics. Due to difficulty of modeling the concrete material behavior, there are some of the studies on the terminal ballistic trajectory of projectiles which has been predicted only by numerical methods [10-16]. For oblique impacts of penetrator, Warren et al. [10, 11] investigated the penetration of steel projectiles at oblique angles. They have been developed a new free surface effect model, based on dynamic spherical cavity-expansion theory, due to the discrepancy between drag forces of projectile acting on the top and bottom sides at oblique angles. The Differential Area Force Law (DAFL) method [12] was proposed to predict the terminal ballistic trajectory of the projectile under the normal stress acting on the projectile which is derived from spherical cavity-expansion theory by Forrestal et al. [5]. $\mathrm{Wu}$ et al. [13] predicted the terminal ballistic trajectory of projectiles considering the mass loss and nose-blunting of ogive-nosed projectiles by using the DAFL method [12] and semi-empirical resistance function, but the CRA was not included. For concrete targets by the compressive and tensile damage model, in order to obtain the CRA, Liu et al. [14] have conducted numerical simulations of oblique angle penetration by using the 3D finite element code LS-DYNA. They presented that the ballistic trajectory at different oblique angles and the ricochet are numerically simulate by using a semi-empirical constitutive model for concrete material. Li et al. [15] discussed the ballistic trajectory and the critical velocity at which the ricochet occurs during the oblique penetration using a semi-empirical forcing function for soil target. Yu et al. [16] computed the ricochet angle for oblique penetration into concrete targets with the spherical cavityexpansion theory and the rigid kinematics, but verification of the numerical result was not presented.

Although there have been proposed some CRA, they focused more on the deformation of projectiles and the implementation of numerical method in finite element code of concrete material constitutive models involving various damage and failure behaviors of concrete targets. In addition, generally, numerical simulation needs much CPU time and it takes a lot of resources to obtain CRA by iterative numerical simulations due to trajectories should be predicted at different oblique angles for the estimation of ricochet. Thus, development of efficient way to obtain CRA, which is one of the major design factors in design strategy, is necessary for warhead design and assessment of warhead performance.

In this study, an efficient computation method to obtain CRA of projectiles into concrete targets is proposed by the spherical cavity-expansion theory and employing a finite differential approach. During the oblique impact events, drag force is approximated by semi-empirical function from the spherical cavity-expansion theory and the finite differential approach, employed in the Differential Area Force Law (DAFL) method [12], is used to predict the projectile motion during the penetration. The semi-empirical model is intended to have accuracy of 3D-finite element method as well as reduce the computing time for the trajectory of projectiles. In order to enhance accuracy of the projectile motion, the empirical constants of the semi-empirical function are obtained by comparing the results of numerical simulations with respect to the oblique angle. CRA is then obtained by predicting the projectile motion according to the oblique angle as well as verified by comparing to the result of finite element simulation. Our work presents that the reliable CRA can be estimated efficiently by employing a series of the numerical simulations.

\section{Numerical Method to Evaluate Trajectory of Oblique Projectiles}

In this paper, CRA is defined as the minimum oblique angle that will produce the projectile starts to bounce back from the surface without penetrating or embedding the target. In order to obtain CRA, projectile motion during penetration into a concrete target is needed to predict accurately at a given oblique angle. The projectile motion is affected by the impact velocity, projectile shape, the oblique angle, and the resistance of the target. In the following section, a numerical method to obtain CRA considering the parameters of the projectile penetration is presented.

2.1. Drag Forces Based on Cavity-Expansion Theory. In order to predict the projectile motion, equations of motions during penetration are established under following assumptions and simplification. First, in the penetration process, the plane of the oblique angle is unchanged and coincides with the plane of the attacking angle which is corresponded to $x-y$ plane as shown in Figure 1. Second, the gravity on the projectile is ignored and the force acting on the projectile is considered as the resistance of the target and the moment of the plane of the oblique angle. Lastly, we allow that the projectile is rigid body which is undergoing translational and rotational motion about fixed axis.

Figure 1 shows initial penetration state of the projectile. Two planar Cartesian coordinates are in the plane of the oblique angle. The origin of the two coordinate systems is located at the center of gravity of the projectile. The $x y$ coordinate indicates global coordinate system, and the $x^{\prime} y^{\prime}$ coordinate indicates local coordinate system which is fixed on the projectile. The $x^{\prime}$-axis and the $y^{\prime}$-axis represent the perpendicular and parallel axis of symmetric axis of projectile, respectively. $\delta$ is the angle of attack which represents the angle between the velocity direction at the center of gravity and the $y^{\prime}$-axis. $\theta$ is the angle between the velocity direction at the center of gravity of the projectile and the $x$-axis;

The normal stress acting on the projectile can be evaluated by the semi-empirical equation derived from the spherical cavity-expansion theory [5]. For semi-infinite concrete 


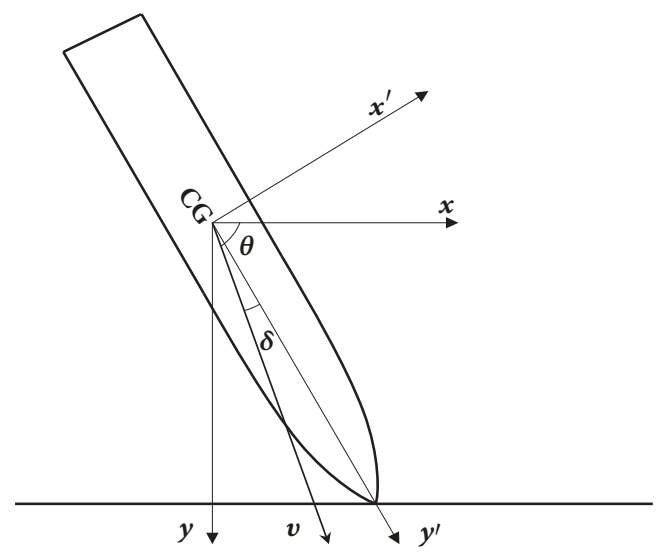

FIgURE 1: A schematic diagram of initial state of the oblique projectile.

targets penetrated by ogive-nosed projectiles, the normal stress is expressed as

$$
\sigma_{n}=S f_{c}^{\prime}+B \rho v_{n}^{2}
$$

where $S$ is the dimensionless empirical constant; $f_{c}^{\prime}$ is the unconfined compressive strength of the concrete material; $\rho$ is density of the concrete material; $v_{n}$ is the expansion velocity of the cavity surface which is identical to the normal velocity of an arbitrary projectile surface, and $B=1$ for concrete targets [4]. The above equation is obtained by regression of the experimental data of normal penetration considering the concrete behavior and friction effect simultaneously under the condition that all parameters but the striking velocity are held constant [5]. According to Anderson Jr. [18] and $\mathrm{Wu}$ et al. [19], the friction effect can be disregarded in penetration mechanics due to the existence of melted layer on the projectile surface that resulted in a frictionless interface. Therefore, the friction effect is not considered in the equation for resistive force analysis.

While a projectile impacts a target obliquely, the asymmetric resistive force, which is induced by the difference between resistive force acting on the top and bottom surface of the projectile, should be considered due to existence of the free surface. Thus, the free surface effect should be taken into account for calculating the resistive force. In this paper, the free surface effect model proposed by Warren et al. [10, 11] is adopted to correct (1). According to the cavity-expansion theory, cavity expands from zero to radius $r_{a}$, which is radius of an arbitrary point in the projectile surface (see Figure 2). The plastic region is located from the radius $r_{a}$ and radius $r_{p}$ which is the distance from the projectile axis to the interface position along a normal line. Similarly, the elastic region is located from the radius $r_{p}$ and radius $r_{d}$ which is distance from the projectile axis to the free surface along a normal line at an arbitrary point in the projectile surface.

The free surface effect model shows that the resistive force acting on the projectile surface depends on the distance to free surface of the target. Warren et al. $[10,11]$ proposed that the normal stress $\left(\sigma_{n}^{*}\right)$, involved in free surface effect, can be expressed as

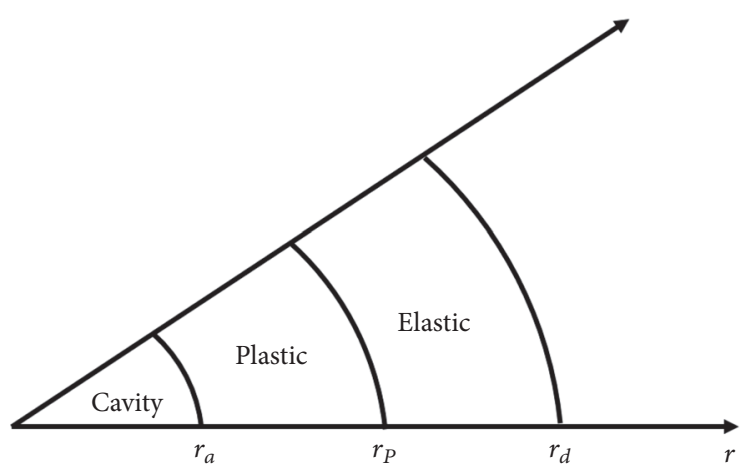

FIGURE 2: Response regions of the plastic-elastic problem in the cavity-expansion model.

$$
\sigma_{n}^{*}=f\left(r_{d}, r_{a}, v\right) \sigma_{n}=f\left(r_{d}, r_{a}, v\right)\left(S f_{c}^{\prime}+B \rho v_{n}^{2}\right)
$$

by establishing a decay function $f\left(r_{d}, r_{a}, v\right)$ to consider the free surface effect. The decay function is expressed as below

$$
f\left(r_{d}, r_{a}, \nu\right)=\frac{\sigma_{n}\left(r_{a}\right)}{\sigma_{n}\left(r_{a}\right)_{r_{d \rightarrow \infty}}}
$$

where $\sigma_{n}\left(r_{a}\right)$ and $\sigma_{n}\left(r_{a}\right)_{r_{d \rightarrow \infty}}$ are the radial stresses at the cavity surface when the free surface effect is considered or not. The decay function takes a value within zero to one. The details of the free surface effect model including equation of the radial stress at the cavity surface are presented in Warren et al. $[10,11]$.

\subsection{Projectile Kinematics during the Oblique Penetration.} During the penetration process, resistive force by concrete target is acting on the projectile surface and this force changes the projectile motion. Figure 3 shows the scheme of resistive force on a longitudinal section of the projectile and cross section across an arbitrary point, respectively [13]. The normal stress, $\sigma_{n}$, is acting on point A with the angle $\alpha$ between the vertical axis of the projectile and the tangential line on point $\mathrm{A}$ where $r$ is the radius of the transverse cross section through point $A ; \beta$ is the angle between the $x^{\prime}$-axis and the projection of $\sigma_{n}$ on the above transverse cross section.

According to Figure 3, $d F_{x^{\prime}}$ and $d F_{y^{\prime}}$, which indicate the resistive forces acting on the $x^{\prime}$ and $y^{\prime}$ directions of the finite element $d S$ at arbitrary point of the projectile surface, respectively, are expressed as follows using the normal stress and the element area of a finite element on the projectile surface.

$$
\begin{aligned}
& d F_{x^{\prime}}=-\sigma_{n} \cos \alpha \cos \beta d S \\
& d F_{y^{\prime}}=-\sigma_{n} \sin \alpha d S
\end{aligned}
$$

The moment about the axis of the plane of oblique angle by the resistive forces acting on the $x^{\prime}$ and $y^{\prime}$ directions of the finite element $d S$ is expressed as follows:

$$
\begin{aligned}
& d M_{x^{\prime}}=y^{\prime} d F_{x^{\prime}} \\
& d M_{y^{\prime}}=-r \cos \beta d F_{y^{\prime}}
\end{aligned}
$$




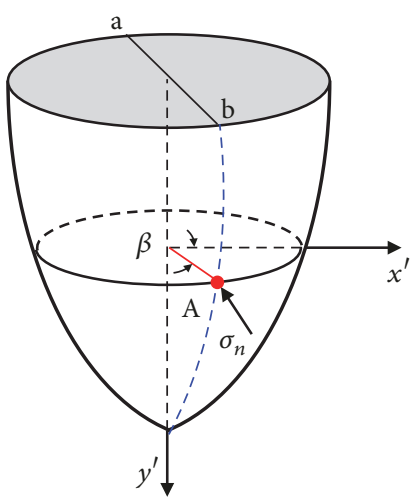

(a)

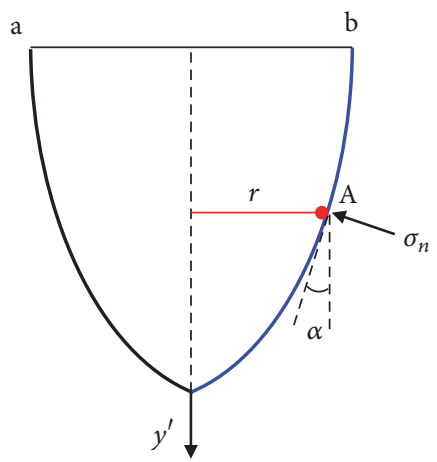

(b)

FIGURE 3: Schematic diagrams of projectile surface acting on resistive force at arbitrary point A [13]. (a) 3D view of projectile acting on normal stress at point $\mathrm{A}$ and (b) view of cross section along the line $\mathrm{ab}$.

Total force and total moment of the projectile are simply calculated as the sum of the forces and moments of each finite element. The equations of motions of the projectile are obtained based on the rigid body kinematics as follows:

$$
\begin{aligned}
m \frac{d^{2} x}{d t^{2}}= & \left(F_{x^{\prime}} \cos \delta-F_{y^{\prime}} \sin \delta\right) \sin \theta \\
& +\left(F_{x^{\prime}} \sin \delta+F_{y^{\prime}} \cos \delta\right) \cos \theta \\
m \frac{d^{2} y}{d t^{2}}= & -\left(F_{x^{\prime}} \cos \delta-F_{y^{\prime}} \sin \delta\right) \cos \theta \\
& +\left(F_{x^{\prime}} \sin \delta+F_{y^{\prime}} \cos \delta\right) \sin \theta \\
I_{0} \varepsilon= & -M
\end{aligned}
$$

where the mass of the projectile is $m ; I_{0}$ is the moment of inertia about the axis of the plane of the oblique angle; $\varepsilon$ is the angular acceleration of the projectile about the axis of the plane of oblique angle.

2.3. Numerical Procedure for Estimation of CRA. We present the flowchart of the analysis procedure for obtaining CRA which is programmed with the MATLAB (see Figure 4). First of all, the projectile surface is discretized into surface elements and characteristics of each element such as element area and direction of element normal are calculated. After judging the penetration of each surface element into the target, the normal stress acting on the penetrated surface element is calculated from (2) and (3). Based on the normal stress and element area, the forces and moments acting on the center of gravity of the projectile are obtained by summation of forces and moments of each surface element. And then the motion parameters such as acceleration, velocity, and rotation angle are calculated by the motion equation from (6) and updated by the Runge-Kutta method at each time step. If the global velocity of the projectile is remained, the motion parameters are updated by the calculation procedure for predicting the projectile until the global velocity equals to zero or the termination time is reached. The occurrence of the ricochet is confirmed by final position of the projectile. In the case of the projectile is embedded or perforated, the final position is set to be positive value with reference to the target surface. In contrast, the position has a negative value for the ricochet. Thus the final position will approach zero at CRA.

The numerical procedure is repeated until the initial oblique angle converges. The converged initial oblique angle is regarded as CRA. A convergence criterion is set to be smaller than a $1 \%$ difference of the initial oblique angle between the previous and current iteration step. If the current initial oblique angle does not meet the convergence criterion, projectile motion calculation with another initial oblique angle is continued. The initial oblique angle for next calculation is determined based on one of the following equations:

$$
\begin{aligned}
& \theta_{n+1}=\frac{\theta_{n}+\theta_{n-1}}{2} \\
& \theta_{n+1}=\theta_{n}-p\left(\theta_{n}\right) \frac{\theta_{n}-\theta_{n-1}}{p\left(\theta_{n}\right)-p\left(\theta_{n-1}\right)}
\end{aligned}
$$

where $\theta_{n}$ is the oblique angle at the $(n)$ th step and $p\left(\theta_{n}\right)$ is the final position of the projectile at the (n)th step. Equation (7) represents the bisection method and (8) represents the secant method for numerical calculation. The numerical efficiency can be evaluated by comparing the CPU time.

\section{Results and Discussion}

3.1. Determination of Empirical Constant of Normal Penetration. In order to predict projectile motion accurately during the penetration into concrete target, the resistive force acting on the projectile surface should be well determined. The empirical constant is necessary to calculate the resistive force based on (2) and (3). The empirical constant can be determined from experimental and numerical data. Li and Chen [20] suggested that the empirical constant $S$ is equal to $S=72 f_{c}^{\prime}{ }_{c}^{-0.5}$ but that value is not correspondent to predict the experimental data conducted by Forrestal et al. [17]. Forrestal et al. [17] concluded that the empirical constant 


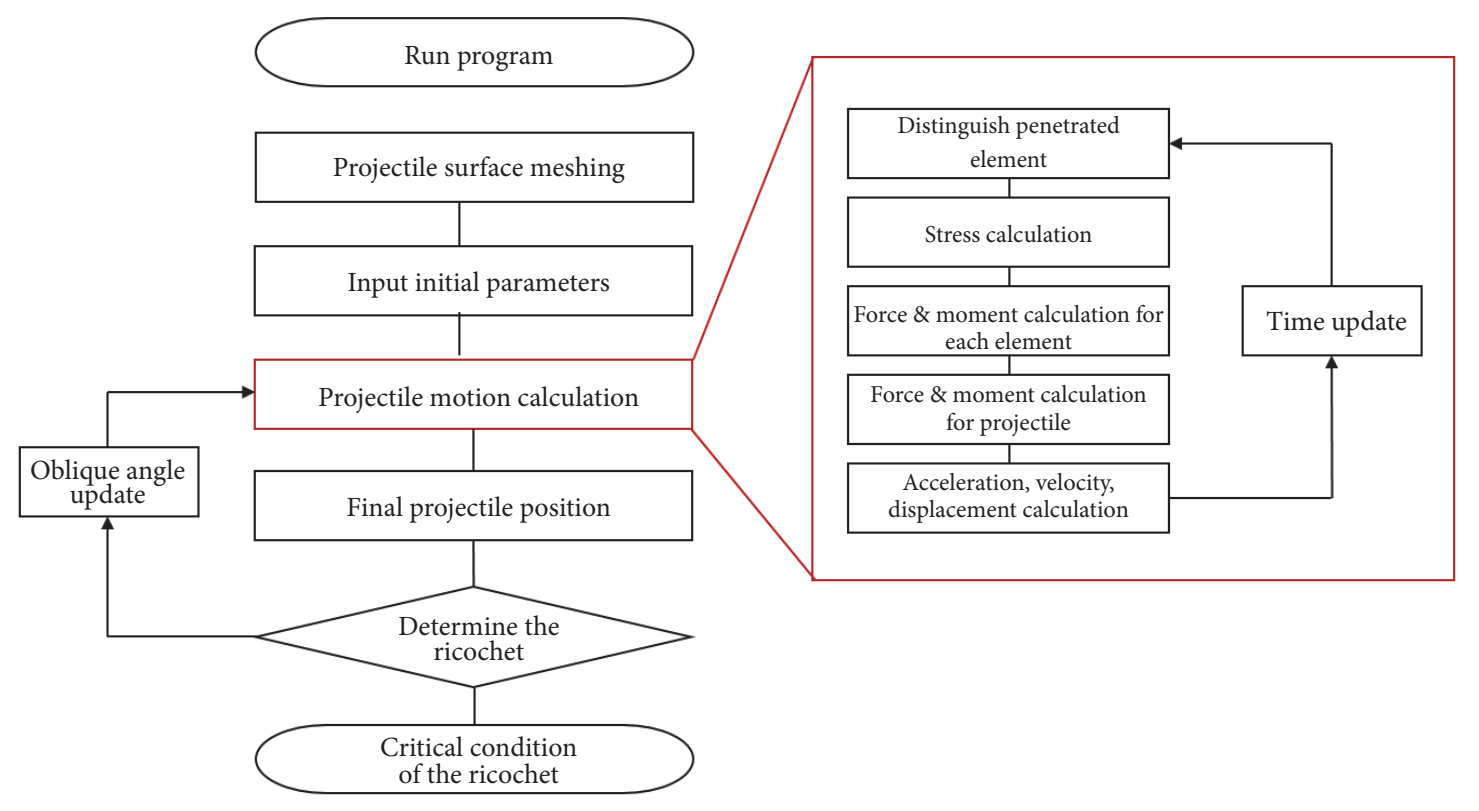

FIGURE 4: Analysis procedure for obtaining CRA.

is different with respect to the experimental condition such as impact velocity and projectile nose shape at the same target. In addition, concrete material shows complicated mechanical behavior according to the loading path [21]. In the case of oblique penetration, normal stress acting on the projectile is asymmetry along the axis of the projectile. The asymmetric stress conditions might effect on the empirical constant of $S$. Thus the empirical constant is necessary to be obtained with various oblique angles.

In this study, for the clarity on the results, the empirical constant for normal penetration is obtained by comparing numerical results of this study with experimental data conducted by Forrestal et al. [17]. Thereby, the accuracy of numerical results can be improved. For the oblique impact simulations, the empirical constants for the oblique penetration with various oblique angles are also estimated by comparing numerical result from this study with results of finite element analysis due to the lack of experimental results. Forrestal et al. conducted normal penetration experiment of small scale projectile into concrete target with various impact velocities [17]. The projectile dimension is shown as Figure 5. The projectile machined by 4340 Rc45 steel has 80 -mm-diameter, 530-mm-length, caliber-radius-head of 3.0 , and mass of $13 \mathrm{~kg}$. The experimental results with various impact velocities are shown in Table 1 . The penetration depth increases with the impact velocity. Generally, penetration depth is dependent on impact velocity and material properties of the target. Since the empirical constant is relevant with resistive normal stress of concrete target, the empirical constant can be determined by predicting penetration depth of normal penetration. In order to predict the penetration depth using the program in this study, the projectile is discretized into 12,000 elements and the time step size of $0.01 \mathrm{~ms}$ is determined from the results of sensitivity analyses

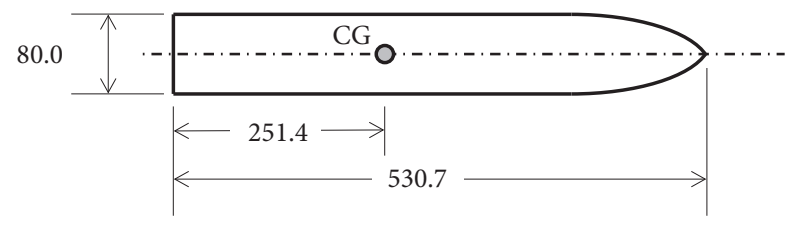

Figure 5: Projectile dimension [17].

in the appendix. The unconfined compressive strength of concrete target is $39 \mathrm{MPa}$ as mentioned in Forrestal et al. [17].

For various impact velocity which ranges from 238.1 to $369.5 \mathrm{~m} / \mathrm{s}$, penetration depths of the projectile are estimated with various empirical constants using the proposed program. The predicted penetration depths obtained by numerical results and experimental results are plotted together in Figure 6 in order to compare the tendency of penetration depth with various velocity. The empirical constant is then obtained using the least square method which best describes the experimental result. We observe that the numerical result with empirical constant of 8.52 shows good agreement with the experimental results.

3.2. Empirical Constant of Oblique Penetration. Due to complicated mechanical behavior of concrete material with various loading angles, the empirical constants are obtained at various oblique angles by comparing with finite element analysis result. Due to the lack of experimental data for the oblique penetration, finite element analyses for the penetration at various oblique angles are conducted to obtain the empirical constants. During the oblique penetration, the projectile motion is more complicated as well as stress state of oblique penetration is more asymmetric than that of normal penetration. Thus, it is necessary to estimate the empirical 
TABLe 1: Penetration data of normal impact tests conducted by Forrestal et al. [17].

\begin{tabular}{lcccc}
\hline Shot and projectile number & Projectile mass $(\mathrm{kg})$ & Striking velocity $(\mathrm{m} / \mathrm{s})$ & Pitch yaw (degrees) & Penetration depth $(\mathrm{m})$ \\
\hline SNL-00-11/3 & 12.923 & 238.1 & $0.1 \mathrm{D} / 0.0$ & 0.30 \\
SNL-00-12/4 & 12.900 & 275.7 & $0.1 \mathrm{D} / 0.4 \mathrm{~L}$ & 0.38 \\
SNL-00-09/1 & 12.910 & 314.0 & $0.2 \mathrm{D} / 0.2 \mathrm{R}$ & 0.45 \\
SNL-00-10/2 & 12.914 & 369.5 & $3.6 \mathrm{U} / 2.5 \mathrm{~L}$ & 0.53 \\
\hline
\end{tabular}

For pitch and yaw, $\mathrm{D}=$ down, $\mathrm{U}=\mathrm{up}, \mathrm{R}=$ right, and $\mathrm{L}=$ left.

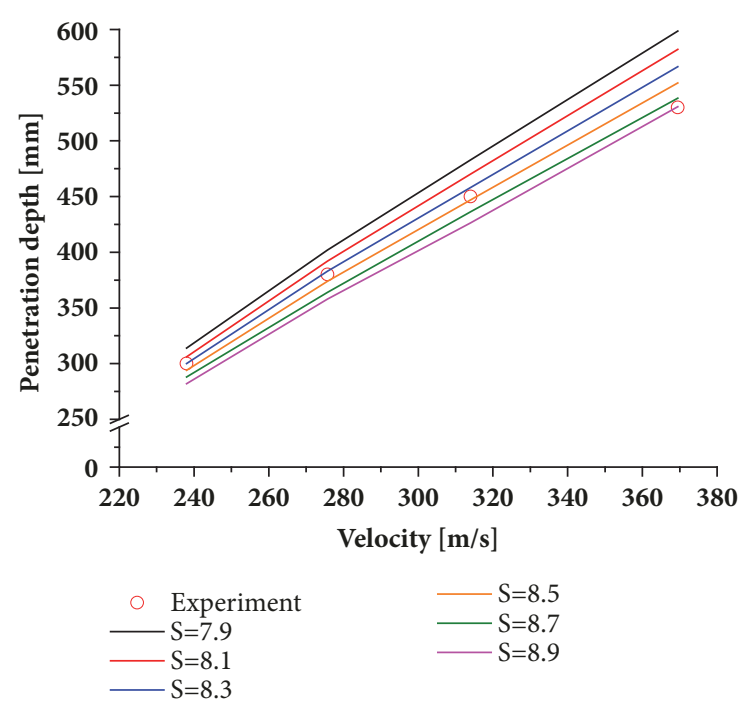

FIgURE 6: Penetration depths with various empirical constants comparing to the experimental result.

constant with respect to the oblique angle at a given impact velocity.

The commercial code LS-Dyna is employed for the finite element analysis. By applying symmetric boundary conditions, half models of the projectile and concrete target are simulated to reduce the computational resource and computing time. Figure 7 shows finite element models for the oblique penetration. The projectile is treated as rigid body and the concrete target is modeled with 8-node reduced integration solid elements of 159,200. For material model for the concrete target, continuous surface cap model proposed by Schwer and Murray [21] and Murray [22] is employed with unconfined compressive strength of $39 \mathrm{MPa}$ and strain independent erosion condition.

Generally, the finite element analysis depends on the element size, the material model and the erosion criterion. The finite element analysis result should be justified before obtaining the empirical constant of oblique penetration. Therefore, the finite element analyses for normal impact tests conducted by Forrestal et al. [17] are conducted first. The numerical results can be justified by comparing the experimental data. Figure 8 shows penetration depth with various striking velocities. It can be shown that the numerical results predict well the experimental data. Therefore, the finite element analysis result is justified.

The oblique angles, which represents as $\theta$, of 15,30 , and 45 degrees with no angle of attack, are simulated in this study

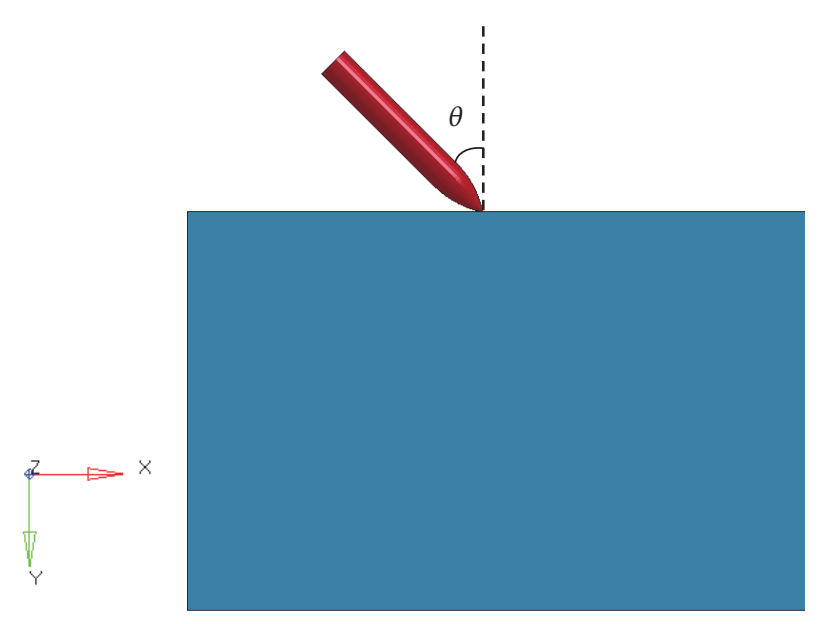

FIGURE 7: Finite element models for oblique penetration.

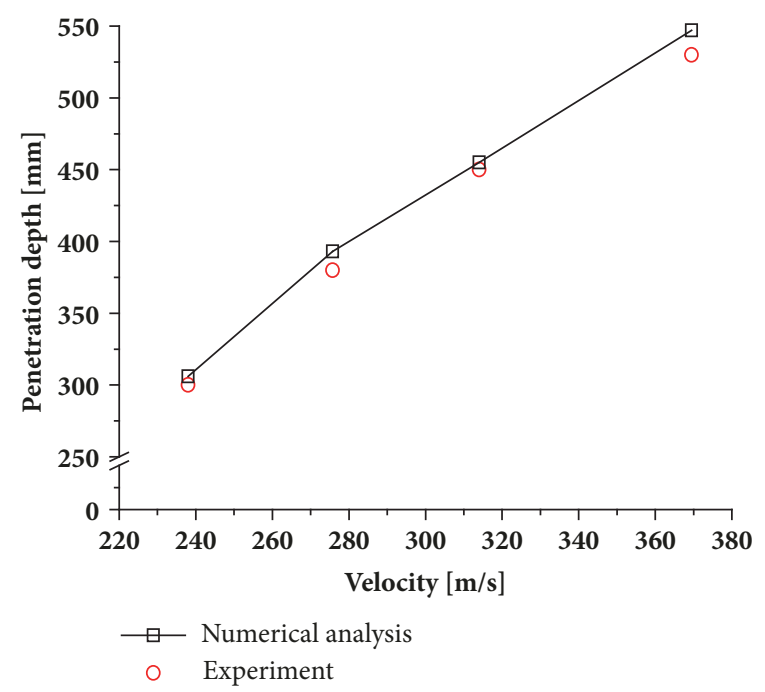

FIgURE 8: Penetration depths with various striking velocities of the numerical results comparing to the experimental data.

that means $\delta$ equals zero in Figure 1 . The projectile impact velocity is chosen to be $238 \mathrm{~m} / \mathrm{s}$. The finite element analysis for oblique penetration is conducted using Intel i7 CPU (4 cores, $3.4 \mathrm{GHz}$ ) and $16 \mathrm{~GB}$ memory. The computation time of single analysis is about 5 hours.

The results of projectile motions during penetration into concrete target with oblique angles of 15, 30, and 45 degrees are shown in Figures 9, 10, and 11, respectively. In order to 


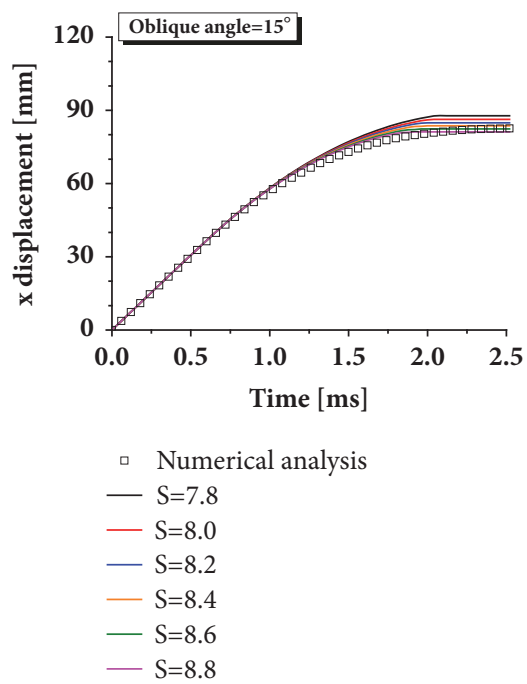

(a)

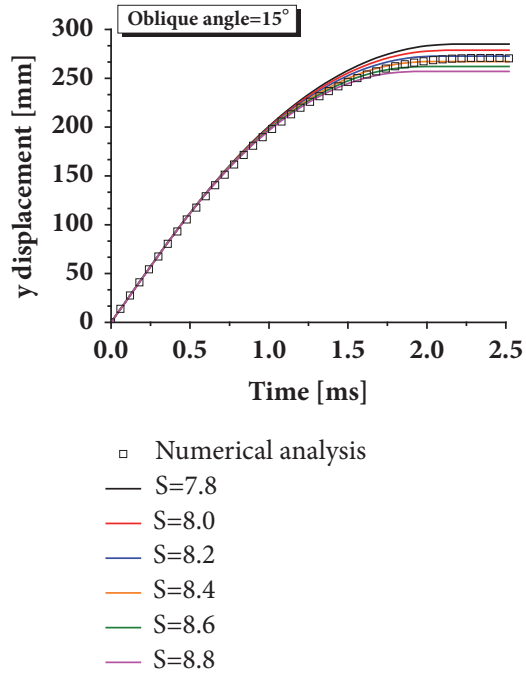

(b)

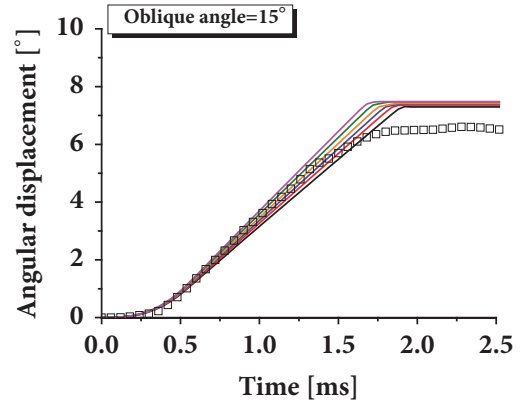

- Numerical analysis

- $\mathrm{S}=7.8$

- $\mathrm{S}=8.0$

- $\mathrm{S}=8.2$

- $\mathrm{S}=8.4$

$-S=8.6$

$-\mathrm{S}=8.8$

FIGURE 9: Projectile motions during the oblique penetration with various empirical constants at the oblique angle of 15 degree: (a) horizontal displacement; (b) vertical displacement; (c) rotation about the axis of plane of oblique angle.

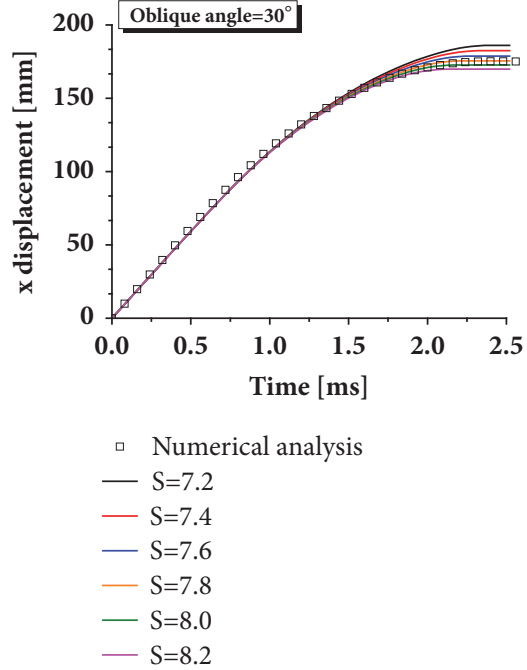

(a)

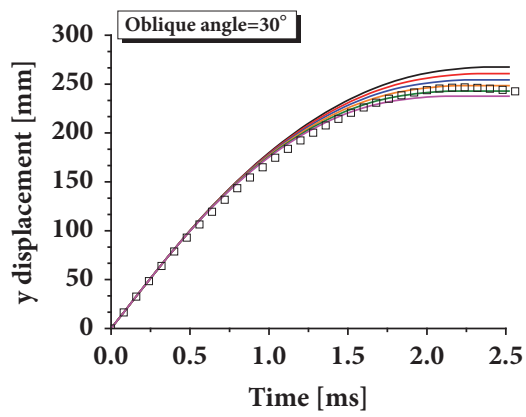

- Numerical analysis

- $\mathrm{S}=7.2$

- $\mathrm{S}=7.4$

- $S=7.6$

- $S=7.8$

- $\mathrm{S}=8.0$

$-\mathrm{S}=8.2$

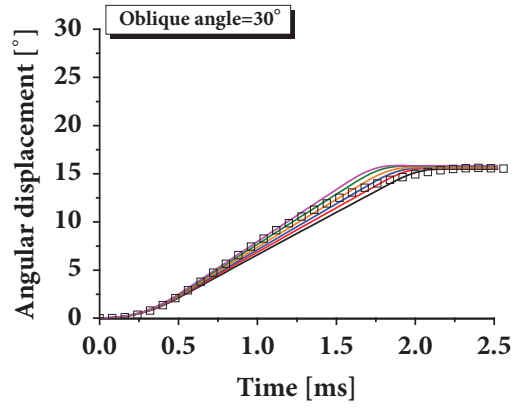

- Numerical analysis

- $\mathrm{S}=7.2$

- $S=7.4$

$-\mathrm{S}=7.6$

$\mathrm{S}=7.8$

- $\mathrm{S}=8.0$

(b)

FIGURE 10: Projectile motions during the oblique penetration with various empirical constants at the oblique angle of 30 degree: (a) horizontal displacement; (b) vertical displacement; (c) rotation about the axis of plane of oblique angle.

obtain the empirical constant at oblique penetration events, we compare the results from the finite element analysis with the results of horizontal, vertical, and angular displacement of the projectile by estimating the proposed numerical procedure. The projectile motion can be predicted differently with various empirical constants. The empirical constants, which best describe the finite element analysis results, were obtained using the least square method mentioned in previous section. The empirical constants with various oblique angles are tabulated in Table 2. We found that the empirical constant decreases with oblique angles. Thus, in order to improve the accuracy of trajectory projectile at the oblique angles the
TABLE 2: Estimated empirical constants of the oblique penetration.

\begin{tabular}{lcccc}
\hline $\begin{array}{l}\text { Oblique angle } \\
\text { (degree) }\end{array}$ & 0 & 15 & 30 & 45 \\
\hline $\begin{array}{l}\text { Empirical } \\
\text { constant }\end{array}$ & 8.52 & 8.25 & 7.91 & 6.48 \\
\hline
\end{tabular}

empirical constant should be considered as a function of the oblique angle.

In order to applying the empirical constants to the developed program with various oblique angles, the empirical constants were fitted by second order polynomial with respect to the oblique angles. Figure 12 shows the empirical constants 


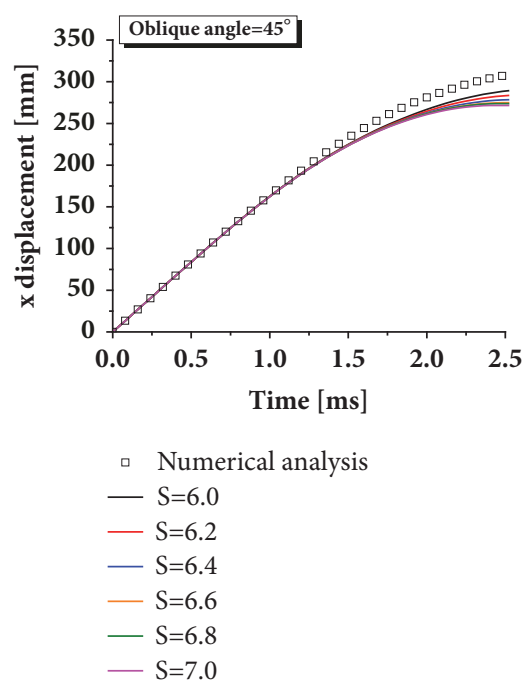

(a)

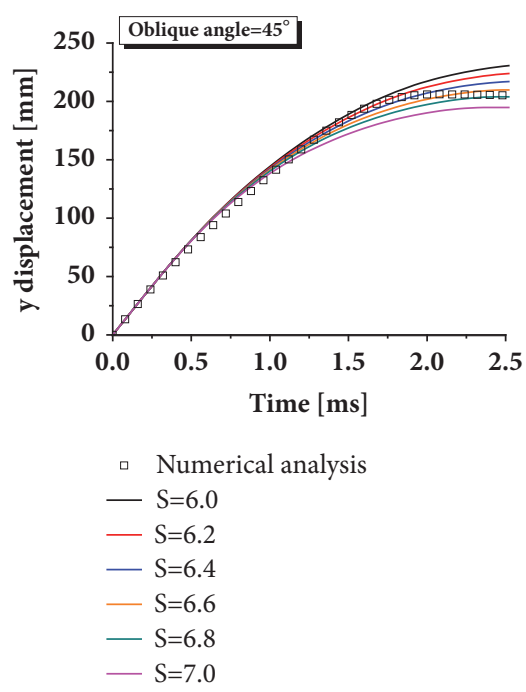

(b)

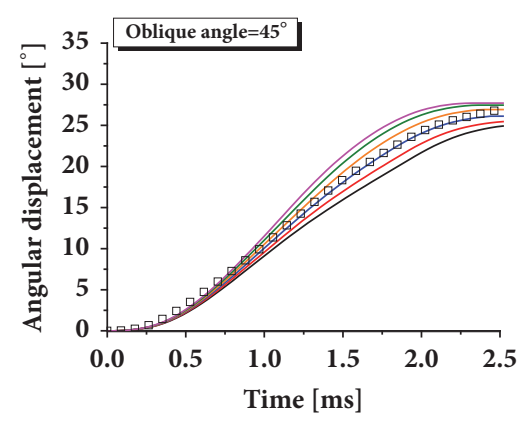

- Numerical analysis

- $\mathrm{S}=6.0$

- $S=6.2$

- $S=6.4$

$-\mathrm{S}=6.6$

$-\mathrm{S}=6.8$

$-S=7.0$

FIGURE 11: Projectile motions during the oblique penetration with various empirical constants at the oblique angle of 45 degree: (a) horizontal displacement; (b) vertical displacement; (c) rotation about the axis of plane of oblique angle.

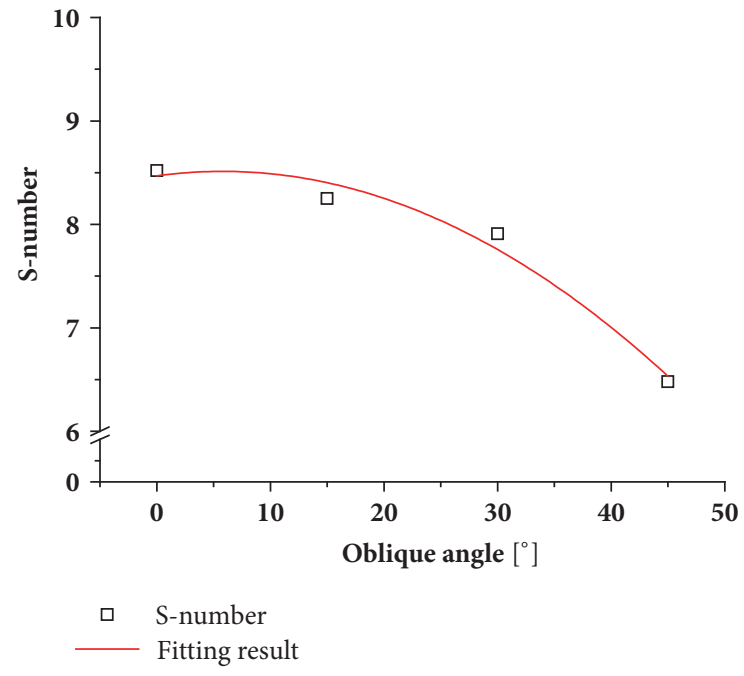

FIGURE 12: Empirical constants with various oblique angles and the fitted data.

with various oblique angles and the fitted data. Regression equation for the empirical constants with various oblique angles is expressed as follows

$$
S(\theta)=\gamma_{0}+\gamma_{1} \theta+\gamma_{2} \theta^{2}
$$

where $\gamma_{0}$ is 8.469; $\gamma_{1}$ is 0.0149 ; and $\gamma_{2}$ is -0.0013 .

\section{Result and Discussion}

According to numerical procedures mentioned in Section 2.3 and Figure 4, the CRA for the projectile is obtained. For analysis conditions, projectile is discretized into 12,000 elements and the unconfined compressive strength of concrete target is $39 \mathrm{MPa}$ as mentioned in previous section. The initial impact velocity is $238 \mathrm{~m} / \mathrm{s}$ and the termination time and time increment are set to be $5 \mathrm{~ms}$ and $0.01 \mathrm{~ms}$, respectively from the results of sensitivity analyses in the appendix,.

In order to evaluate numerical efficiency, the bisection method and the secant method expressed in (7) and (8) are employed to find the CRA which meets the convergence criteria in the oblique angle range of 40 to 60 degree. Figures 13 and 14 show the input oblique angle and the projectile position at $3.2 \mathrm{~ms}$ after impact with respect to numerical iteration number for both methods, respectively. In the Figure 13, solid point and hollow point represent the condition where the ricochet occurs or not, respectively. We decide that the ricochet occurs when the final position of projectile is located above the target surface (see Figure 14). For both numerical methods, the initial oblique angle converges to CRA as iteration number increases. We observe that the initial oblique angle converges faster when the secant method is used than when the bisection method is used. As shown in Figure 13, the CRA for the projectile is obtained as the oblique angle of 48.2 degree.

In order to verify the result of the estimated CRA, finite element analyses of the oblique penetration are conducted at least 5 cases with different initial oblique angles based on the result using secant method. We approximate the CRA in the oblique angle range of 40 to 60 degree. Figure 15 shows projectile motion during penetration at the CRA resulted from the program in this study and the finite element analysis. The projectile motion at the CRA resulted from the program well approximates the result from the finite element analysis. It is concluded that the CRA is obtained accurately and reliably.

Numerical efficiency for obtaining the minimum oblique angle, led to the ricochet of the projectile, is evaluated by comparing CPU time for the program in this study and the finite element analysis of the oblique penetration. 


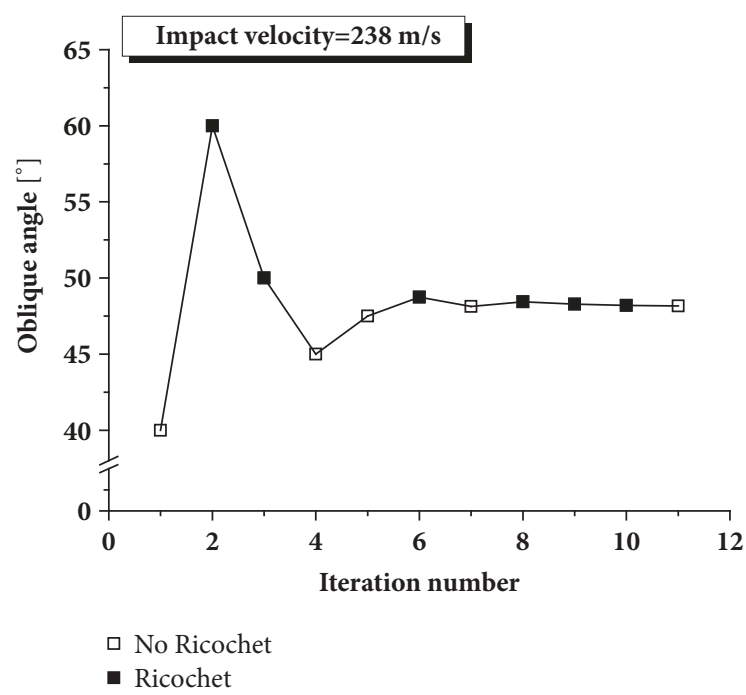

(a)

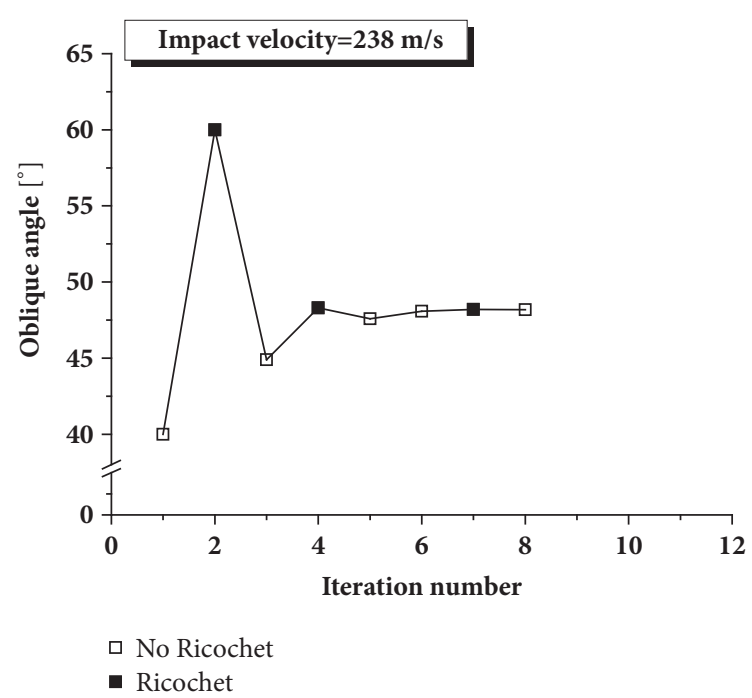

(b)

FIGURE 13: Initial oblique angles with respect to numerical iteration number for (a) the bisection method; (b) the secant method.

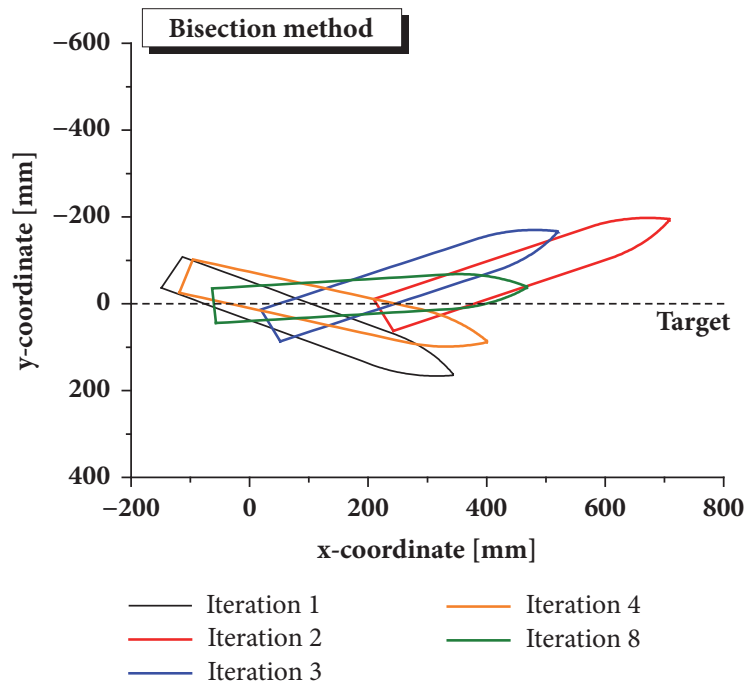

(a)

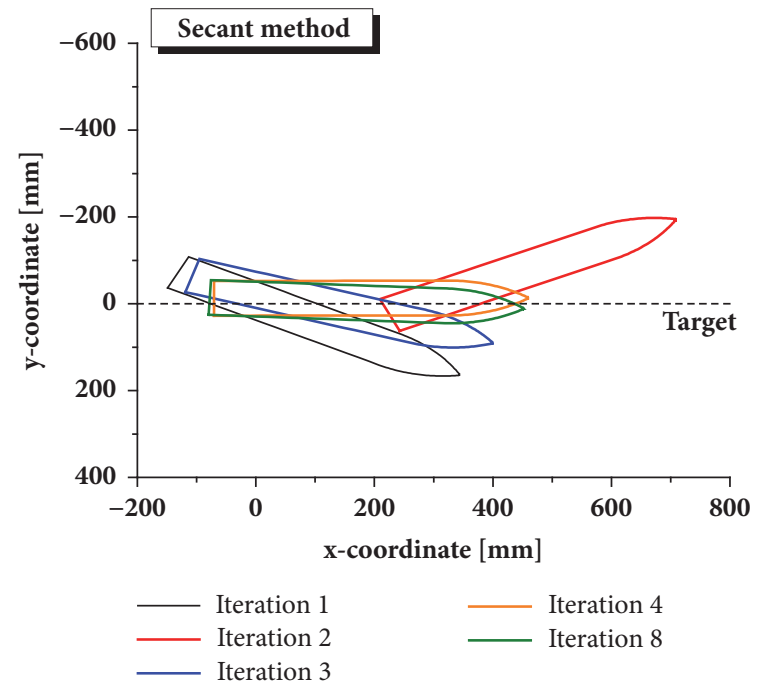

(b)

FIGURE 14: Projectile position at $3.2 \mathrm{~ms}$ after impact with respect to numerical iteration number for (a) the bisection method; (b) the secant method.

Figure 16 shows normalized CPU time to obtain the CRA. The proposed program with the secant method needs the least CPU time and much faster than that of the finite element analysis. The CRA can be estimated accurately and efficiently by the proposed program.

\section{Conclusion}

This paper proposed an efficient computation method to obtain CRA for the oblique penetration into the concrete target based on the spherical cavity-expansion theory and a finite differential approach. The CRA can be estimated by predicting projectile motion during the oblique penetration simulations with various oblique angles. The projectile motion is predicted by a finite differential approach and semiempirical method by using resistance derived from spherical cavity-expansion theory. In order to enhance the projectile motion, the empirical constant of the semi-empirical resistance function is obtained as well as fitted by conducting finite element analysis with various oblique angles. Then the CRA is obtained and verified with the finite element analysis result. Numerical efficiency is also evaluated by root-finding algorithms and comparing the finite element analysis. From the result, it is concluded that accurate CRA can be obtained efficiently.

Extending this study, the ricochet behavior with various influential factors should be addressed in the future. Since projectile can penetrate a concrete target in many different 


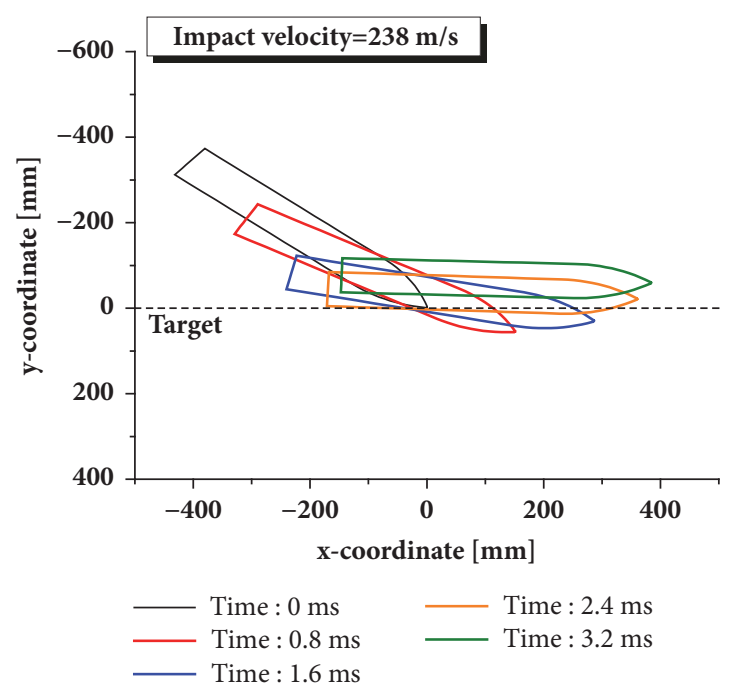

(a)

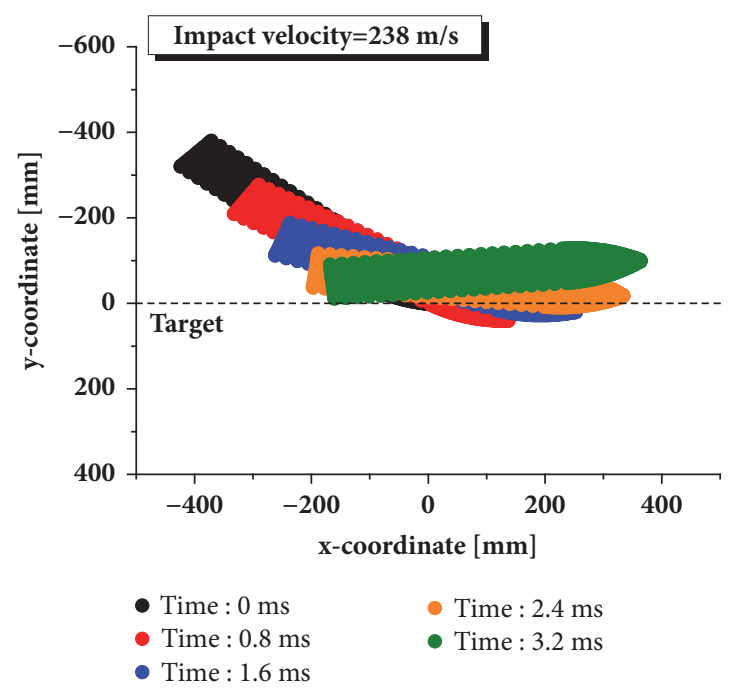

(b)

FIGURE 15: Projectile motion during the oblique penetration at the CRA, estimated as 48.2 degree, resulted from (a) the proposed program in this study; (b) the finite element analysis.

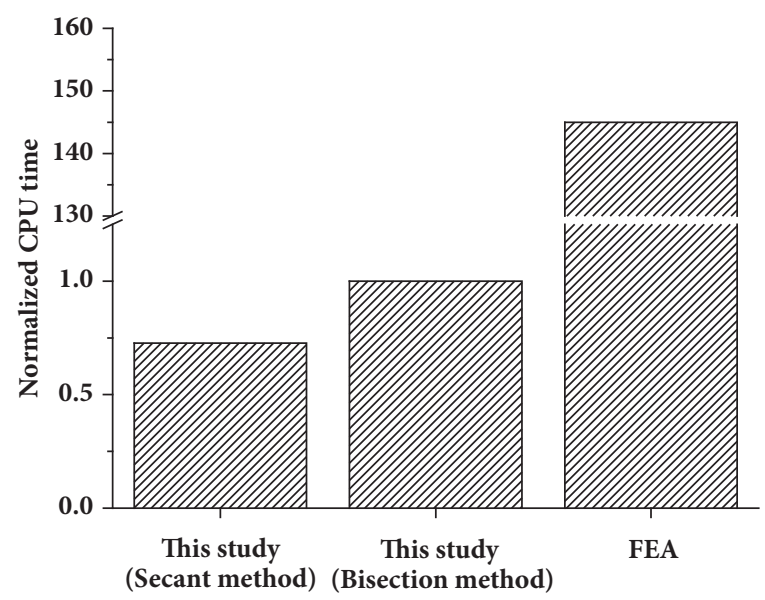

Figure 16: Normalized CPU time to obtain the CRA.

scenarios, it is essential to perform comprehensive analysis of the ricochet behavior. For estimation of the CRA of penetrator warhead, we suggest that this numerical platform will become a very useful and powerful tool as well as help to design the penetrator warhead. Additionally, a design of the ricochet optimized projectile can be further developed.

\section{Appendix}

The number of elements and the time step size used in this paper are the values where the calculation results converge. In order to show the convergence of the calculation result according to the number of elements and the time step size, the sensitivity analyses are conducted for the developed program at the oblique angle of 45 degree. Figure 17 shows projectile motions during the oblique penetration with various time step sizes when the number of elements is kept constant as 12,000 . It is shown that the time step size can influence the projectile trajectory but the calculated results start to converge at the time step size of $0.01 \mathrm{~ms}$. Figure 18 shows projectile motions during the oblique penetration depending on the number of projectile elements at constant time step size of $0.01 \mathrm{~ms}$. It is shown that the number of elements can influence the projectile trajectory but the sensitivity is not great.

\section{Data Availability}

The data used to support the findings of this study are included within the article. 


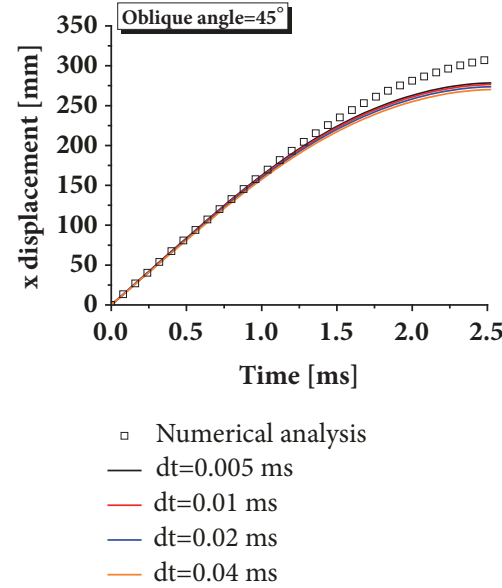

(a)

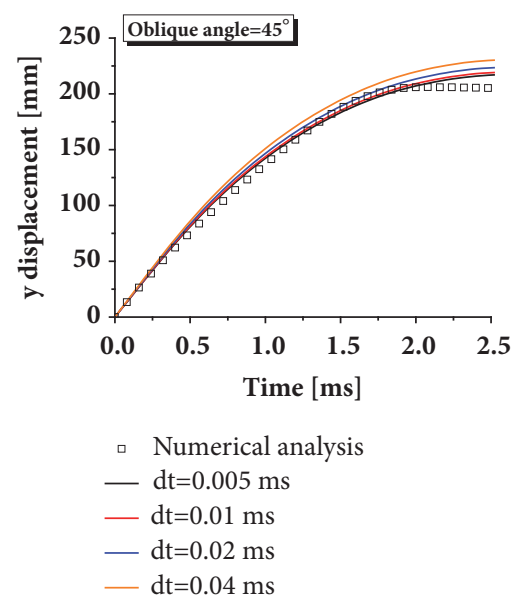

(b)

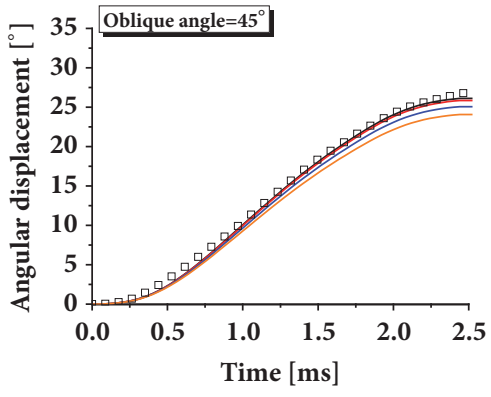

- Numerical analysis

- $\mathrm{dt}=0.005 \mathrm{~ms}$

- $\mathrm{dt}=0.01 \mathrm{~ms}$

- $\mathrm{dt}=0.02 \mathrm{~ms}$

- dt $=0.04 \mathrm{~ms}$

(c)

FIGURE 17: Projectile motions during the oblique penetration with various time step sizes at the oblique angle of 45 degree: (a) horizontal displacement; (b) vertical displacement; (c) rotation about the axis of plane of oblique angle.

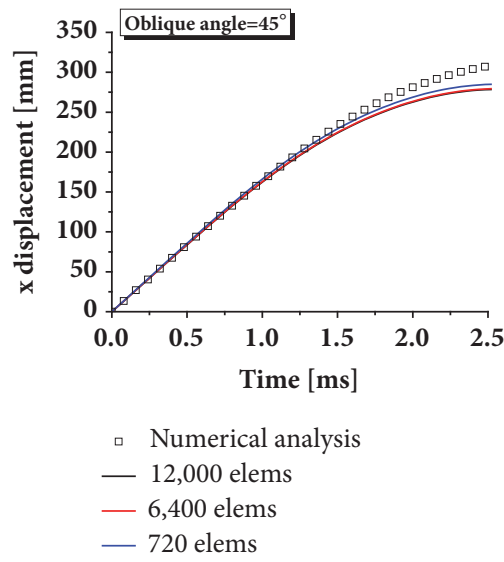

(a)

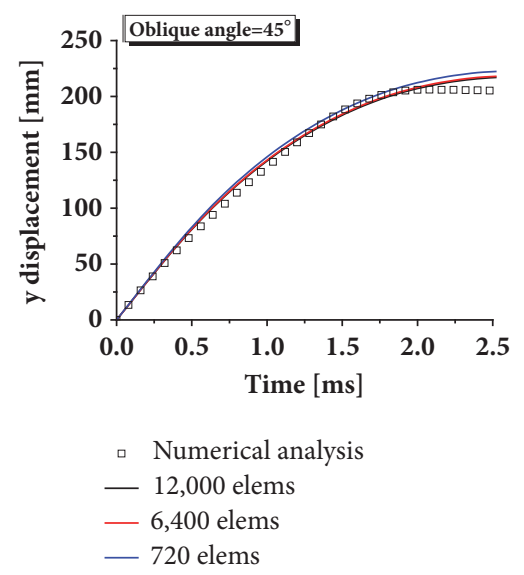

(b)

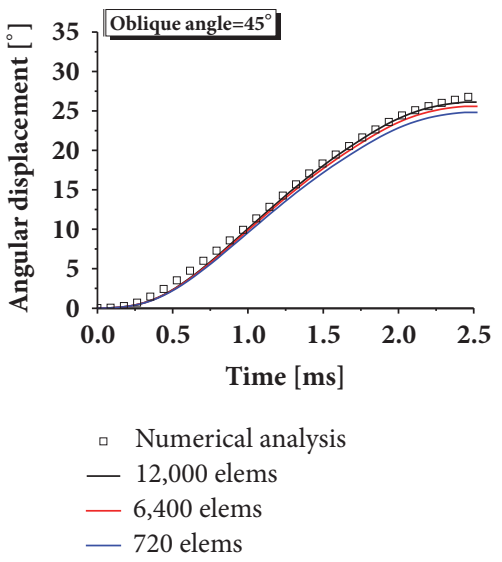

(c)

FIGURE 18: Projectile motions during the oblique penetration depending on the number of elements at the oblique angle of 45 degree: (a) horizontal displacement; (b) vertical displacement; (c) rotation about the axis of plane of oblique angle.

\section{Conflicts of Interest}

The authors declare that they have no conflicts of interest.

\section{Acknowledgments}

The work was supported by the Agency for Defense Development, Republic of Korea, and research funds for newly appointed professors of Chonbuk National University in 2018.

\section{References}

[1] S. J. Hanchak, M. J. Forrestal, E. R. Young, and J. Q. Ehrgott, "Perforation of concrete slabs with $48 \mathrm{MPa}$ (7 ksi) and $140 \mathrm{MPa}$ (20 ksi) unconfined compressive strengths," International Journal of Impact Engineering, vol. 12, no. 1, pp. 1-74, 1992.
[2] A. N. Dancygier and D. Z. Yankelevsky, "High strength concrete response to hard projectile impact," International Journal of Impact Engineering, vol. 18, no. 6, pp. 583-599, 1996.

[3] A. N. Dancygier and D. Z. Yankelevsky, "Penetration mechanisms of non-deforming projectiles into reinforced concrete barriers," Structural Engineering and Mechanics, vol. 13, no. 2, pp. 171-186, 2002.

[4] M. J. Forrestal, B. S. Altman, J. D. Cargile, and S. J. Hanchak, "An empirical equation for penetration depth of ogive-nose projectiles into concrete targets," International Journal of Impact Engineering, vol. 15, no. 4, pp. 395-405, 1994.

[5] M. J. Forrestal and D. Y. Tzou, "A spherical cavity-expansion penetration model for concrete targets," International Journal of Solids and Structures, vol. 34, no. 31-32, pp. 4127-4146, 1997.

[6] A. Tate, "A simple estimate of the minimum target obliquity required for the ricochet of a high speed long rod projectile," Journal of Physics D: Applied Physics, vol. 12, no. 11, pp. 18251829, 1979. 
[7] Z. Rosenberg, Y. Yeshurun, and M. Mayseless, "On the Ricochet of long rod projectiles," in Proceedings of the 11th International Symposium of Ballistics, p. 501, 1989.

[8] K. Daneshjou and M. Shahravi, "Penetrator strength effect in long-rod critical ricochet angle," Journal of Mechanical Science and Technology, vol. 22, no. 11, pp. 2076-2089, 2008.

[9] E. J. A. T. Mattijssen, K. D. H. Pater, and R. D. Stoel, "Ricochet behavior on glass-critical ricochet angles, ricochet angles, and deflection angles," Journal of Forensic Sciences, vol. 61, no. 6, pp. 1456-1460, 2016.

[10] T. L. Warren and K. L. Poormon, "Penetration of 6061-T6511 aluminum targets by ogive-nosed VAR 4340 steel projectiles at oblique angles: Experiments and simulations," International Journal of Impact Engineering, vol. 25, no. 10, pp. 993-1022, 2001.

[11] T. L. Warren, S. J. Hanchak, and K. L. Poormon, "Penetration of limestone targets by ogive-nosed VAR 4340 steel projectiles at oblique angles: Experiments and simulations," International Journal of Impact Engineering, vol. 30, no. 10, pp. 1307-1331, 2004.

[12] F. E. Heuze, "An over review of projectile penetration into geological materials with emphasis on rocks," International Journal of Rock Mechanics and Mining Sciences, vol. 27, pp. 1-14, 1990.

[13] H. Wu, X. W. Chen, Q. Fang, X. Z. Kong, and L. L. He, "Stability analyses of the mass abrasive projectile high-speed penetrating into a concrete target Part III: Terminal ballistic trajectory analyses," Acta Mechanica Sinica, vol. 31, no. 4, pp. 558-569, 2015.

[14] Y. Liu, F. Huang, and A. Ma, "Numerical simulations of oblique penetration into reinforced concrete targets," Computers \& Mathematics with Applications, vol. 61, no. 8, pp. 2168-2171, 2011.

[15] Q. M. Li and E. A. Flores-Johnson, "Hard projectile penetration and trajectory stability," International Journal of Impact Engineering, vol. 38, no. 10, pp. 815-823, 2011.

[16] W. Yu, T. Wang, and L. Dong, "Computation of ricochet angle for oblique penetration of warhead into concrete target," Journal of Projectiles, Rockets, Missiles and Guidance, vol. 28, no. 5, pp. 109-118, 2008 (Chinese).

[17] M. J. Forrestal, D. J. Frew, J. P. Hickerson, and T. A. Rohwer, "Penetration of concrete targets with deceleration-time measurements," International Journal of Impact Engineering, vol. 28, no. 5, pp. 479-497, 2003.

[18] C. E. Anderson Jr., "Analytical models for penetration mechanics: A Review," International Journal of Impact Engineering, vol. 108, pp. 3-26, 2017.

[19] H. Wu, X.-W. Chen, L.-L. He, and Q. Fang, "Stability analyses of the mass abrasive projectile high-speed penetrating into concrete target. Part I: Engineering model for the mass loss and nose-blunting of ogive-nosed projectiles," Acta Mechanica Sinica, vol. 30, no. 6, pp. 933-942, 2014.

[20] Q. M. Li and X. W. Chen, "Dimensionless formulae for penetration depth of concrete target impacted by a non-deformable projectile," International Journal of Impact Engineering, vol. 28, no. 1, pp. 93-116, 2003.

[21] L. E. Schwer and Y. D. Murray, "A three-invariant smooth cap model with mixed hardening," International Journal for Numerical and Analytical Methods in Geomechanics, vol. 18, no. 10, pp. 657-688, 1994.

[22] Y. D. Murray, User Manual for LS-Dyna Concrete Material Model 159, FHWA-HRT-05-062, 2007. 


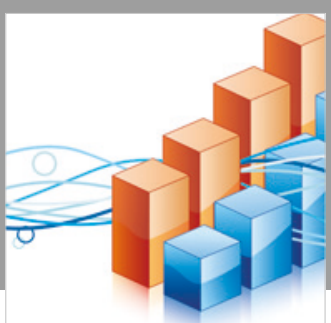

Advances in

Operations Research

\section{-n-m}
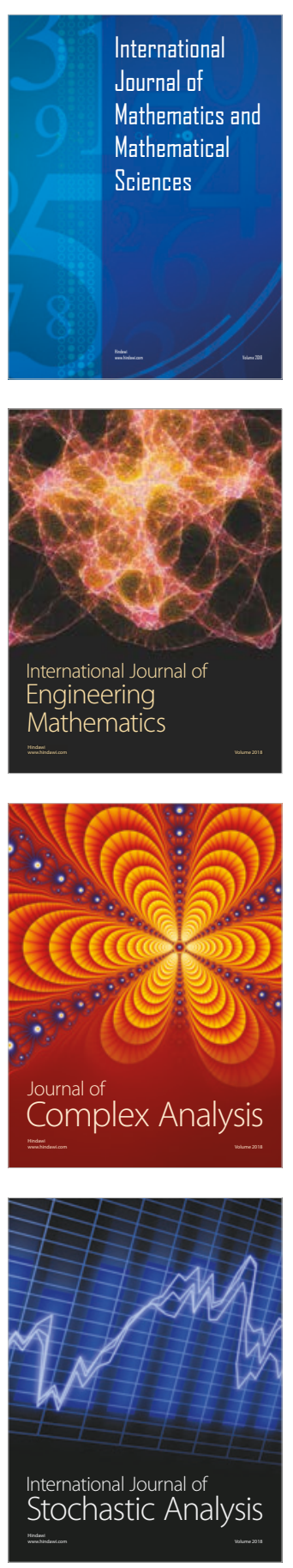
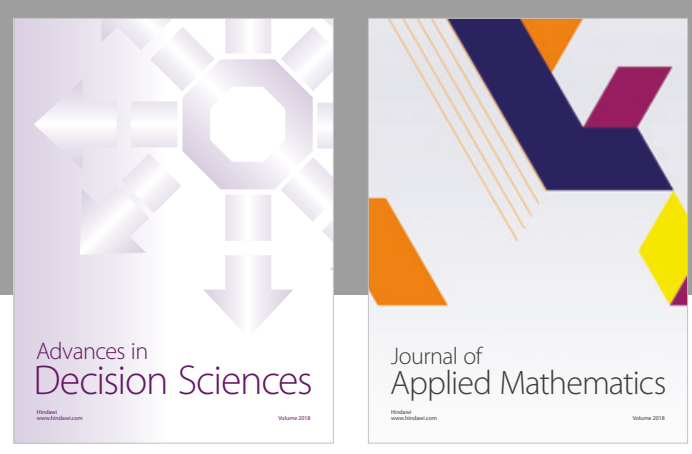

Journal of

Applied Mathematics
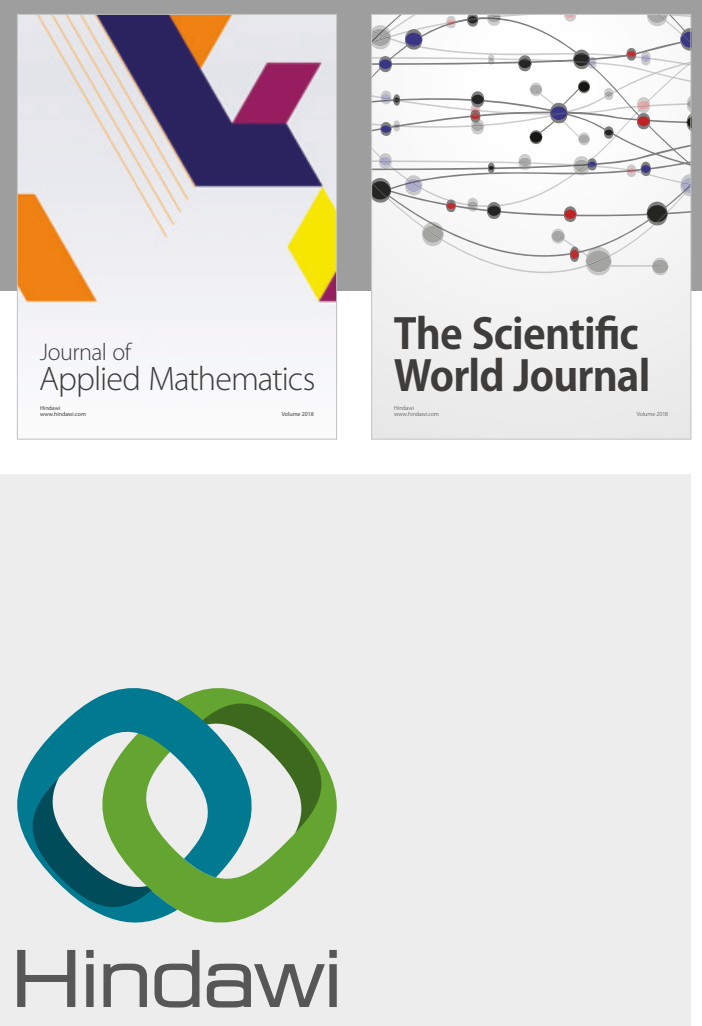

Submit your manuscripts at

www.hindawi.com

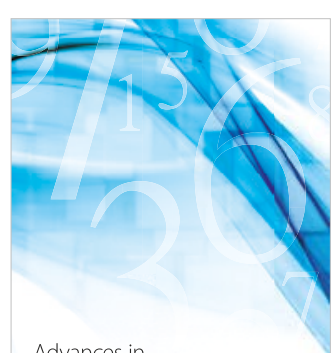

Advances in
Numerical Analysis
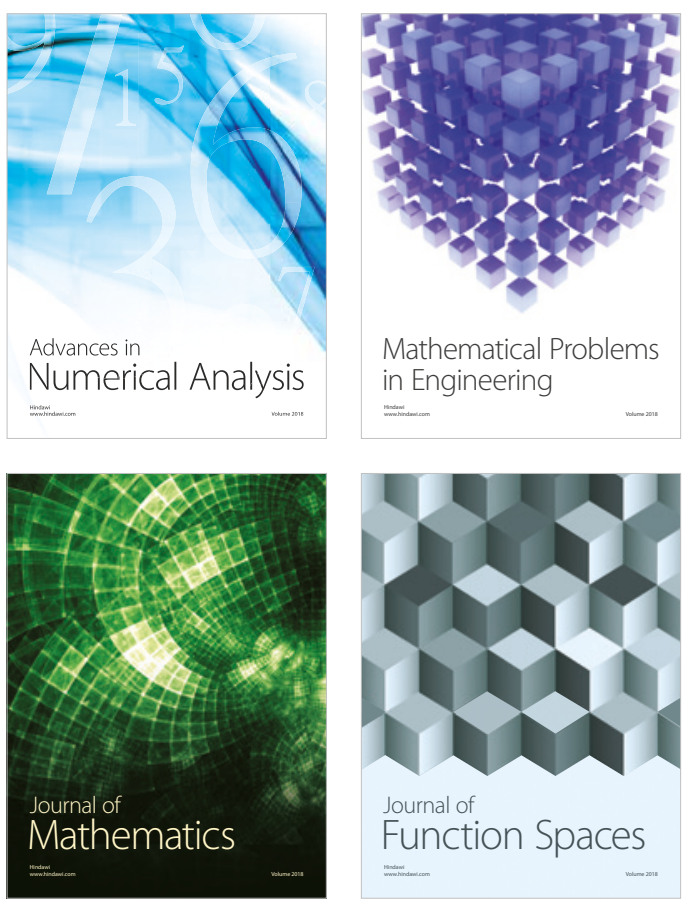

Mathematical Problems in Engineering

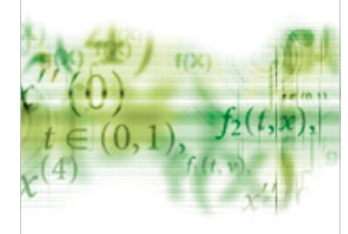

International Journal of

Differential Equations

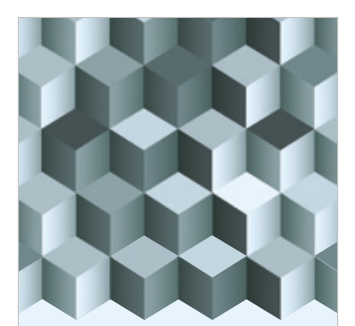

Journal of

Function Spaces
The Scientific

World Journal

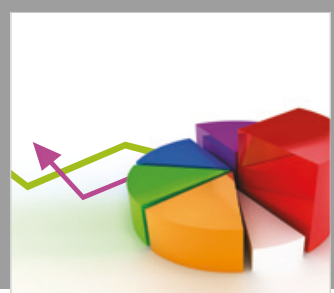

Journal of

Probability and Statistics
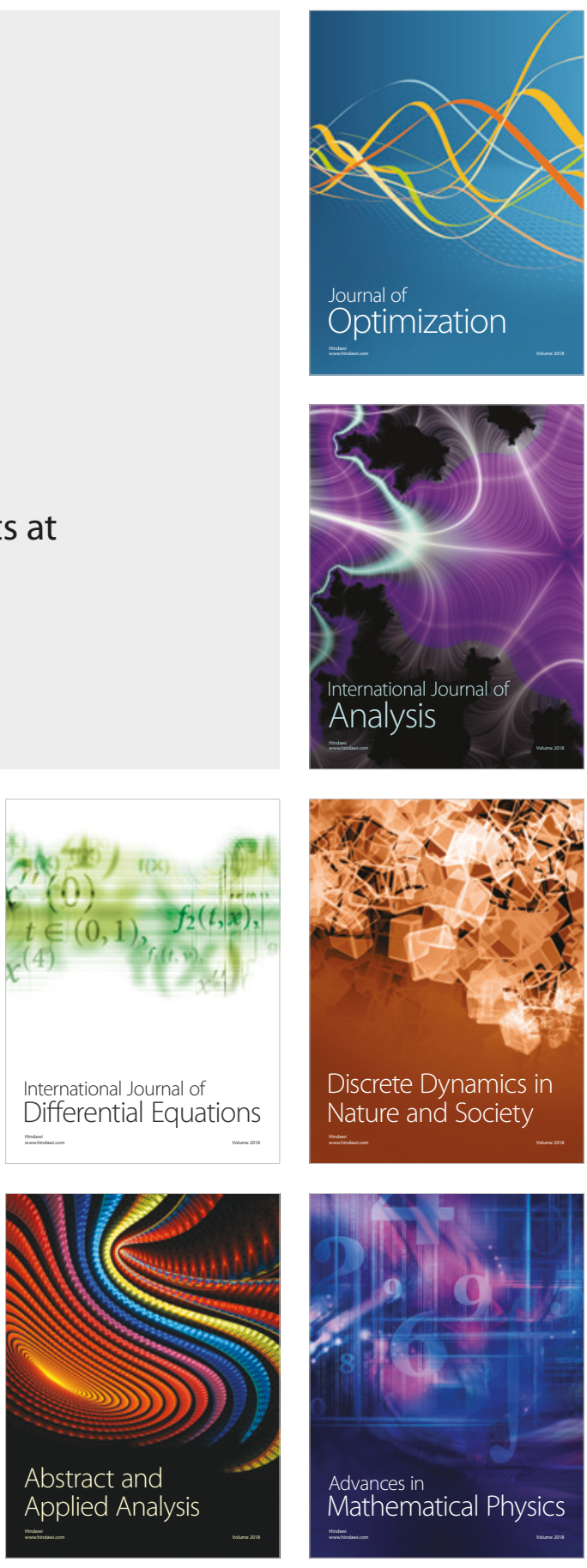